\title{
ESCAPE: An Open-Label Trial of Personalized Immunotherapy in Critically III COVID-19 Patients
}

\author{
Eleni Karakike ${ }^{a}$ George N. Dalekos ${ }^{b}$ loannis Koutsodimitropoulos ${ }^{c}$ Maria Saridaki $^{a}$
}

Chryssa Pourzitaki $^{d}$ Georgios Papathanakos ${ }^{\mathrm{e}}$ Antigone Kotsaki $^{\mathrm{a}}$ Stamatios Chalvatzis ${ }^{\mathrm{a}}$ Vasiliki Dimakopoulou $^{f}$ Nikolaos Vechlidis $^{\text {a }}$ Elisabeth Paramythiotou ${ }^{g}$ Christina Avgoustou $^{\text {a }}$ Aikaterini loakeimidou $^{\mathrm{h}}$ Elli Kouriannidi ${ }^{\mathrm{a}}$ Apostolos Komnos $^{i}$ Evangelia Neou $^{\mathrm{i}}$ Nikoletta Rovina ${ }^{j}$ Eleni Stefanatou ${ }^{c}$ Haralampos Milionis ${ }^{k}$ George Nikolaidis ${ }^{h}$ Antonia Koutsoukou ${ }^{j}$ Georgia Damoraki ${ }^{a}$ George Dimopoulos ${ }^{g}$ Vassileios Zoumpos ${ }^{a}$ Jesper Eugen-Olsen' Karolina Akinosoglou ${ }^{f}$ Nikolaos K. Gatselis ${ }^{b}$ Vasilios Koulouras ${ }^{e}$ Eleni Gkekad Nikolaos Markouc Mihai G. Netea ${ }^{\mathrm{m}, \mathrm{n}}$ Evangelos J. Giamarellos-Bourboulis ${ }^{\mathrm{a}}$

${ }^{a}$ Fourth Department of Internal Medicine, National and Kapodistrian University of Athens, Medical School, Athens, Greece; ${ }^{b}$ Department of Medicine and Research Laboratory of Internal Medicine, National Expertise Center of Greece in Autoimmune Liver Diseases, General University Hospital of Larissa, Larissa, Greece; 'Intensive Care Unit of Latseion Burn Center, General Hospital of Eleusis Thriasion, Eleusis, Greece; dIntensive Care Unit, AHEPA Thessaloniki General Hospital, Thessaloniki, Greece; 'Department of Critical Care Medicine, University of loannina, School of Health Sciences, Faculty of Medicine, loannina, Greece; ${ }^{f}$ Department of Internal Medicine, University of Patras, Medical School, Rion, Greece; ${ }^{9}$ Second Department of Critical Care Medicine, National and Kapodistrian University of Athens, Medical School, Athens, Greece; ' Intensive Care Unit, Asklipeion General Hospital, Voula, Greece; 'Intensive Care Unit, Koutlimpaneion-Triantafylleion Larissa General Hospital, Larissa, Greece; ${ }^{j}$ First Department of Pulmonary Medicine and Intensive Care Unit, National and Kapodistrian University of Athens, Medical School, Athens, Greece; ${ }^{\text {kFirst }}$ Department of Internal Medicine, University of loannina, School of Health Sciences, Faculty of Medicine, loannina, Greece; 'Clinical Research Centre, Copenhagen University Hospital Hvidovre, Hvidovre, Denmark; 'mepartment of Internal Medicine and Center for Infectious Diseases, Radboud University, Nijmegen, The Netherlands; ${ }^{n}$ Immunology and Metabolism, Life \& Medical Sciences Institute, University of Bonn, Bonn, Germany

\section{Keywords}

Interleukin 1 receptor antagonist protein - Tocilizumab . Respiratory distress syndrome · COVID-19 - Macrophage activation - Monocytes

Trial Registration: ClinicalTrials.gov, NCT04339712.

\begin{abstract}
Background: Macrophage activation-like syndrome (MALS) and complex immune dysregulation (CID) often underlie acute respiratory distress (ARDS) in COVID-19. We aimed to investigate the effect of personalized immunotherapy on clinical improvement of critical COVID-19. Methods: In this open-label prospective trial, 102 patients with ARDS by
\end{abstract}

(C) 2021 The Author(s).

Published by S. Karger AG, Basel

This is an Open Access article licensed under the Creative Common Attribution-NonCommercial-4.0 International License (CC BY-NC) (http://www.karger.com/Services/OpenAccessLicense), applicable to the online version of the article only. Usage and distribution for commercial purposes requires written permission.
Correspondence to:

Evangelos J. Giamarellos-Bourboulis, egiamarel@med.uoa.gr 
SARS-CoV-2 were screened for MALS (ferritin $>4,420 \mathrm{ng} / \mathrm{mL}$ ) and CID (ferritin $\leq 4,420 \mathrm{ng} / \mathrm{mL}$ and low human leukocyte antigen (HLA)-DR expression on CD14-monocytes). Patients with MALS or CID with increased aminotransferases received intravenous anakinra; those with CID and normal aminotransferases received tocilizumab. The primary outcome was $\geq 25 \%$ decrease in the Sequential Organ Failure Assessment (SOFA) score and/or $50 \%$ increase in the respiratory ratio by day 8 ; 28 -day mortality, change of SOFA score by day 28 , serum biomarkers, and cytokine production by mononuclear cells were secondary endpoints. Results: The primary study endpoint was met in $58.3 \%$ of anakinra-treated patients and in $33.3 \%$ of tocilizumab-treated patients ( $p: 0.01$ ). Most patients in both groups received dexamethasone as standard of care. No differences were found in secondary outcomes, mortality, and SOFA score changes. Ferritin decreased among anakinra-treated patients; interleukin- 6 , soluble urokinase plasminogen activator receptor, and HLA-DR expression increased among tocilizumab-treated patients. Survivors by day 28 who received anakinra were distributed to lower severity levels of the WHO clinical progression scale. Greater incidence of secondary infections was found with tocilizumab treatment. Conclusion: Immune assessment resulted in favorable anakinra responses among critically ill patients with COVID-19 and features of MALS.

\section{(C) 2021 The Author(s)}

Published by S. Karger AG, Basel

\section{Background}

Early from the start of the pandemic caused by SARS$\mathrm{CoV}-2$, it was realized that patients with severe or critical illness present with profound immune dysregulation with the main features of lymphopenia, hyperproduction of pro-inflammatory cytokines, hyperferritinemia, and pro-inflammatory/anti-inflammatory imbalance [1-6]. An important question arising regarding the transition from severe COVID-19 pneumonia to critical illness and acute respiratory distress syndrome (ARDS) was whether this was identical in all patients, or whether different immune endotypes can be described in the severely ill patients [7]. Based on earlier studies, we used serum ferritin and the expression of the human leukocyte antigen (HLA)-DR on circulating monocytes as biomarkers of immune dysregulation. Ferritin has emerged as a biomarker of COVID-19, indicative of hypercytokinemia, hyperinflammation, and cell damage resulting from sustained macrophage activation; hyperferritinemia is also indicative of liver dysfunction, pro-coagulation, and substantial risk for death $[5,6]$. We selected a cutoff ferritin of $4,420 \mathrm{ng} / \mathrm{mL}$ for the diagnosis of macrophage activation-like syndrome (MALS), as previously done for sepsis $[8,9]$. Analysis showed that almost $25 \%$ of patients with ARDS by COVID-19 had MALS, whereas the rest had low expression of HLA-DR on circulating monocytes. This low HLA-DR expression was interpreted as complex immune dysregulation (CID), since circulating monocytes retained their potential for cytokine production [7].

Previous studies in sepsis have shown that patients with MALS have survival benefit from treatment with anakinra, a nonglycosylated recombinant protein of interleukin (IL)-1 receptor antagonist [10]. The addition of the IL-6 receptor antagonist tocilizumab in cultures of circulating monocytes of patients with ARDS by COVID-19 and CID restored the expression of HLA-DR [7]. These findings guided the concept to approach immunotherapy in critically ill patients with COVID-19 through personalized treatment based on their immune profile, that is, MALS or CID. Efficiency in management of organ dysfunction associated with infection by the novel SARS$\mathrm{CoV}-2$ virus through a personalized immunotherapy approach (ESCAPE) was an exploratory phase II trial where patients with critical COVID-19 were allocated to openlabel treatment with anakinra or tocilizumab if they were presenting with MALS or CID, respectively.

\section{Patients and Methods}

ESCAPE was an open-label phase II prospective clinical trial conducted in 4 departments of internal medicine and 7 intensive care units of tertiary hospitals in Greece between April 2020 and November 2020 (EudraCT number 2020-001039-29; Clinicaltrials.gov NCT04339712). The trial was conducted in accordance with the Declaration of Helsinki and was approved by the National Ethics Committee of Greece (approval 30/20) and by the National Organization for Medicines of Greece (approval IS 021-20).

Enrolled patients were adults of both genders with written informed consent provided by themselves or their legal representatives; they presented ARDS and laboratory findings of MALS or CID. MALS was defined as any serum ferritin $>4,420 \mathrm{ng} / \mathrm{mL}$. CID was defined as a serum ferritin $\leq 4,420 \mathrm{ng} / \mathrm{mL}$ and $<5,000$ receptors of the membrane HLA-DR or 30 mean fluorescence intensity units of HLA-DR on blood CD14-monocytes by flow cytometry (methods details are shown in the online suppl. material; for all online suppl. material, see www.karger.com/doi/10.1159/000519090). Exclusion criteria were stage IV malignancy; do not resuscitate decision; active tuberculosis; infection by the human immunodeficiency virus; primary immunodeficiencies; intake of corticosteroids for more 15 days; anti-cytokine biological treatments the last 1 month; history of systemic lupus erythematosus or demyelinating disorder; and pregnancy or lactation. 
Fig. 1. Study flow of patients in the ESCAPE trial. ALT, alanine aminotransferase; AST, aspartate aminotransferase; ITT, intent-to-treat; LRTI, lower respiratory tract infection; MALS, macrophage activation-like syndrome; PLT, absolute platelet count; PMNs, absolute neutrophil count.

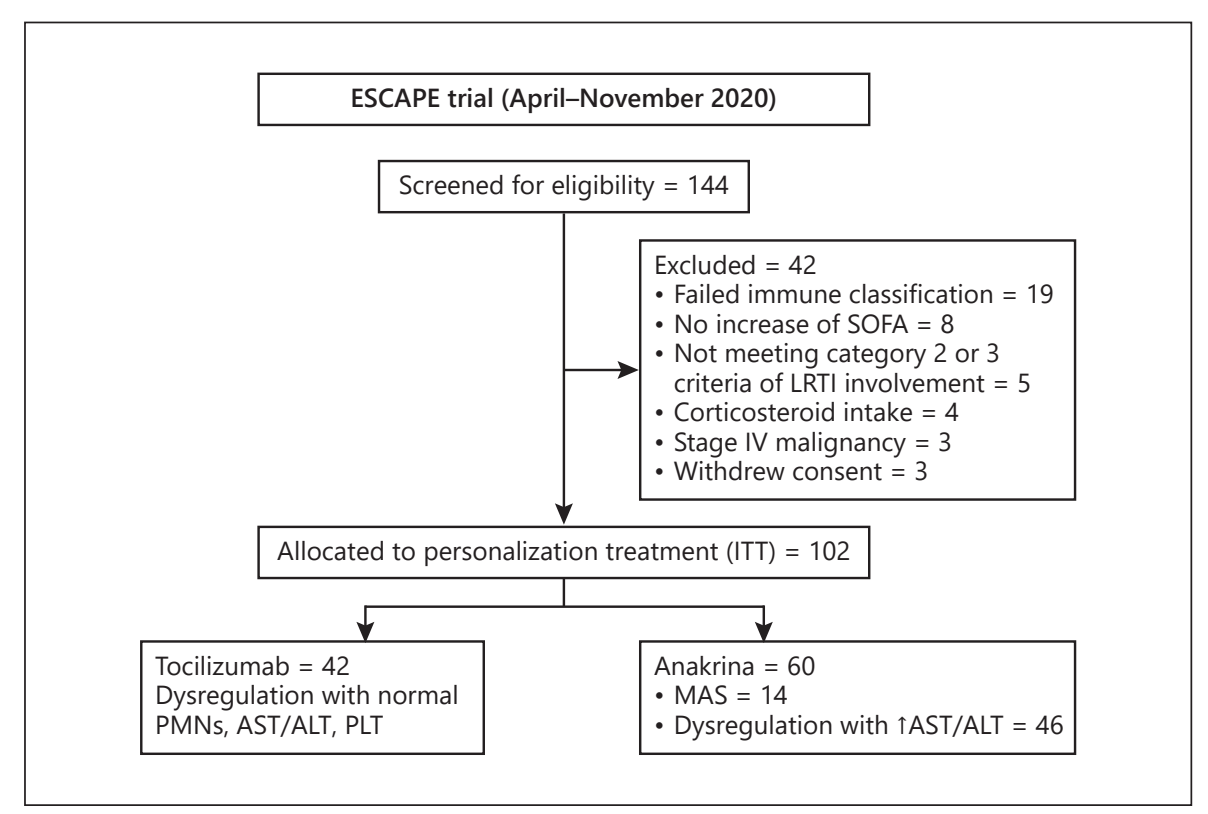

Patients were treated with anakinra for MALS and with tocilizumab for CID. Patients with CID and a neutrophil count $<2,500$ / $\mathrm{mm}^{3}$ or a platelet count $<100,000 / \mathrm{mm}^{3}$ or serum aminotransferases $>1.5 \times$ the upper normal when tocilizumab is contraindicated were treated with anakinra. Although anakinra is not the best suitable candidate drug for these patients, the need for some biological treatment other than tocilizumab, which is not allowed due to the hematological profile and/or liver function and the moderate features of macrophage activation present in CID, has driven the decision to treat them with anakinra. Both drugs were administered intravenously: tocilizumab as single $8 \mathrm{mg} / \mathrm{kg}$ dose up to $800 \mathrm{mg}$ maximum and anakinra $200 \mathrm{mg}$ every $8 \mathrm{~h}$ for 7 days; anakinra was adjusted to $100 \mathrm{mg}$ every $8 \mathrm{~h}$ for 7 days for patients with a creatinine clearance $<30 \mathrm{~mL} / \mathrm{min}$. Immunotherapy was initiated as soon as possible after the results of the immune function assessment became known to the study investigators. Standard-of-care treatment was decided by the attending physicians. Peripheral blood mononuclear cells (PBMCs) were isolated at baseline and on day 4 and stimulated for cytokine production. Patients were followed up daily; adverse events were captured (online suppl. material).

The primary study endpoint was any $25 \%$ or more decrease in baseline Sequential Organ Failure Assessment (SOFA) score and/or at least $50 \%$ increase in the baseline $\mathrm{PaO}_{2} / \mathrm{FiO}_{2}$ ratio by day 8 . Secondary study endpoints were 28-day mortality, change of the SOFA score by day 28 , and changes of serum biomarkers and of cytokine production by PBMCs. Exploratory endpoints were the WHO clinical progression scale (CPS) [11] and the length of hospital stay.

As potential comparators without immunotherapy, we assessed all admissions with ARDS at 3 other intensive care units of Greek tertiary hospitals, during the same time period (online suppl. material). Concurrent comparators were finally selected from a database of 136 patients after applying the inclusion and exclusion criteria of the ESCAPE trial, with the exception of the laboratory criteria for CID, which were not available (online suppl. Fig. 1, provided in the online suppl. material).
The primary endpoint was compared between anakinra- and tocilizumab-treated patients by Fisher's exact test and further validated by forward stepwise logistic regression analysis. Biomarkers and cytokines were expressed as means $\pm \mathrm{SE}$; serial comparisons were done by Wilcoxon's paired test and correlations according to Spearman's rank of order. The distribution of the WHO CPS was compared by Pearson's $\chi^{2}$ test. The time to hospital discharge was compared by the log-rank test. Any value of $p$ below 0.05 was considered significant. The study was exploratory and no power calculation was done.

\section{Results}

The first patient was enrolled on April 2nd, 2020, and the last patient on November 16th, 2020. A total of 102 patients were enrolled; 42 were allocated to treatment with tocilizumab and 60 to treatment with anakinra. Among anakinra-treated patients, 14 had MALS and 46 had CID with increased aminotransferases (Fig. 1). No differences were found between patients allocated to anakinra treatment and to tocilizumab treatment regarding their baseline characteristics (Table 1).

The primary study endpoint was met in $58.3 \%$ of anakinra-treated patients and in $33.3 \%$ of tocilizumabtreated patients (Table 2). This difference was confirmed after stepwise forward logistic regression analysis (OR 3.11; 95\% CIs 1.29-7.73; p: 0.01) (Table 3). However, no difference was found between the 2 groups regarding 28 day mortality and the change of SOFA score by day 28 (Table 2). Response to anakinra for patients with MALS and for patients with CID and hematological/liver dys- 
Table 1. Baseline characteristics of patients enrolled in ESCAPE trial

\begin{tabular}{|c|c|c|c|}
\hline & $\begin{array}{l}\text { Anakinra } \\
(N=60)\end{array}$ & $\begin{array}{l}\text { Tocilizumab } \\
(N=42)\end{array}$ & $p$ value \\
\hline \multicolumn{4}{|l|}{ Age } \\
\hline Mean (SD), years & $61.6(16.4)$ & $66.3(10.8)$ & 0.13 \\
\hline$>65$ years, $n(\%)$ & $28(46.7)$ & $25(59.5)$ & 0.28 \\
\hline$\leq 65$ years, $n(\%)$ & $32(53.3)$ & $17(40.5)$ & \\
\hline \multicolumn{4}{|l|}{ Sex, $n(\%)$} \\
\hline Female & $15(25.0)$ & $8(19.0)$ & \multirow{2}{*}{0.63} \\
\hline Male & $45(75.0)$ & $34(81.0)$ & \\
\hline Ethnicity (white), $n(\%)$ & $60(100)$ & $42(100)$ & 1.00 \\
\hline Invasive mechanical ventilation, $n(\%)$ & $39(65.0)$ & $32(76.2)$ & 0.28 \\
\hline \multicolumn{4}{|l|}{ Severity scores, mean (SD) } \\
\hline $\mathrm{CCl}$ & $2.63(2.38)$ & $2.72(1.48)$ & 0.83 \\
\hline PSI & $82.4(32.9)$ & $89.1(29.6)$ & 0.31 \\
\hline APACHE II score & $10.3(7.9)$ & $11.3(11.3)$ & 0.61 \\
\hline SOFA score & $4.42(2.31)$ & $4.74(2.06)$ & 0.48 \\
\hline \multicolumn{4}{|l|}{ Comorbidities, $n$ (\%) } \\
\hline Type 2 diabetes mellitus & $9(15.0)$ & $10(23.8)$ & 0.31 \\
\hline Chronic heart failure & $3(5.0)$ & $1(2.4)$ & 0.64 \\
\hline Chronic renal disease & $3(5.0)$ & $1(2.4)$ & 0.64 \\
\hline Coronary heart disease & $8(13.3)$ & $7(16.7)$ & 0.78 \\
\hline Arterial hypertension & $12(20.0)$ & $20(47.6)$ & 0.005 \\
\hline Cerebrovascular disease & $2(3.3)$ & $0(0.0)$ & 0.51 \\
\hline COPD & $3(5.0)$ & $1(2.4)$ & 0.64 \\
\hline Atrial fibrillation & $5(8.3)$ & $1(2.4)$ & 0.40 \\
\hline Dyslipidemia & $12(20.0)$ & $14(33.3)$ & 0.17 \\
\hline Hypothyroidism & $5(8.3)$ & $2(4.8)$ & 0.70 \\
\hline \multicolumn{4}{|l|}{ Laboratory values, mean (SD) } \\
\hline Absolute neutrophil count, $/ \mathrm{mm}^{3}$ & $7,284.6(4,605.1)$ & $9,392.0(5,501.3)$ & 0.06 \\
\hline Absolute lymphocyte count, $/ \mathrm{mm}^{3}$ & $879.5(624.8)$ & $835.0(693.4)$ & 0.78 \\
\hline Platelets, $\times 10^{3} / \mathrm{mm}^{3}$ & $238.6(107.8)$ & $281.9(96.1)$ & 0.06 \\
\hline AST, U/L & $61.5(39.7)$ & $36.4(15.9)$ & $<0.0001$ \\
\hline $\mathrm{ALT}, \mathrm{U} / \mathrm{L}$ & $65.7(48.6)$ & $31.9(13.9)$ & $<0.0001$ \\
\hline Creatinine, $\mathrm{mg} / \mathrm{dL}$ & $1.03(0.51)$ & $0.87(0.25)$ & 0.13 \\
\hline \multicolumn{4}{|l|}{ Treatment, $n(\%)$} \\
\hline$\beta$-lactamase inhibitor & $13(21.7)$ & $4(9.5)$ & 0.18 \\
\hline Piperacillin/tazobactam & $24(40.0)$ & $17(40.5)$ & 1.00 \\
\hline 3rd generation cephalosporin & $21(35.0)$ & $10(23.8)$ & 0.28 \\
\hline Ceftaroline & $16(26.7)$ & $15(35.7)$ & 0.38 \\
\hline Ceftazidime/avibactam & $5(8.3)$ & $8(19.1)$ & 0.14 \\
\hline Colistin & $25(41.7)$ & $23(54.8)$ & 0.23 \\
\hline Meropenem & $22(36.7)$ & $22(52.4)$ & 0.16 \\
\hline Glycopeptide & $11(18.3)$ & $17(40.5)$ & 0.02 \\
\hline Linezolid & $26(43.3)$ & $24(57.1)$ & 0.23 \\
\hline Tigecycline & $14(23.3)$ & $17(40.5)$ & 0.08 \\
\hline Moxifloxacin & $5(8.3)$ & $11(26.2)$ & 0.03 \\
\hline Azithromycin & $38(63.3)$ & $19(45.2)$ & 0.11 \\
\hline Hydroxychloroquine & $9(15.0)$ & $9(21.4)$ & 0.44 \\
\hline Remdesivir & $17(28.3)$ & $14(33.3)$ & 0.66 \\
\hline Dexamethasone & $35(58.3)$ & $30(71.4)$ & 0.21 \\
\hline
\end{tabular}

APACHE II, Acute Physiology and Chronic Health Evaluation; AST, aspartate aminotransferase; ALT, alanine aminotransferase; $\mathrm{CCl}$, Charlson's Comorbidity Index; COPD, chronic obstructive pulmonary disease; $n$, number; SOFA, sequential organ failure assessment; SD, standard deviation; SOFA, Sequential Organ Failure Assessment Score; PSI, Pneumonia Severity Index. Bold text denotes significance at $<0.05$ level. 
Table 2. Primary and secondary endpoints of the ESCAPE trial

\begin{tabular}{lllll}
\hline & Anakinra $(n=60)$ & Tocilizumab $(n=42)$ & OR $(95 \% \mathrm{Cl})$ & $p$ value \\
\hline Primary endpoint, $n(\%)(95 \% \mathrm{Cl})$ & $35(58.3 ; 44.9-70.7)$ & $14(33.3 ; 20.0-49.6)$ & $2.80(1.23-6.37)$ & $\mathbf{0 . 0 2}$ \\
At least $25 \%$ decrease of baseline $\mathrm{SOFA}$ score by day 8 & $23(38.3 ; 26.4-51.8)$ & $7(16.7 ; 7.5-32.0)$ & $3.11(1.19-8.15)$ & $\mathbf{0 . 0 3}$ \\
At least $50 \%$ increase of the baseline $\mathrm{PaO}_{2} / \mathrm{FiO}_{2}$ ratio by day 8 & $18(30.0 ; 19.2-43.4)$ & $12(28.6 ; 16.2-44.8)$ & $1.07(0.45-2.55)$ & 1.00 \\
28-day mortality, $n(\%)(95 \% \mathrm{Cl})$ & $20(33.3 ; 22.0-46.8)$ & $14(33.3 ; 20.0-49.6)$ & $1.00(0.43-2.31)$ & 1.00 \\
Delta SOFA day 28, median $(\mathrm{Q} 1-\mathrm{Q} 3)$ & $-10.0(-75$ to 257$)$ & $0(-55$ to 440$)$ & $\mathrm{NA}$ & 0.32 \\
\hline
\end{tabular}

$\mathrm{Cl}$, confidence interval; NA, nonapplicable; OR, odds ratio; Q, quartile; SOFA, Sequential Organ Failure Assessment Score. Bold text indicates significance at 0.05 level.

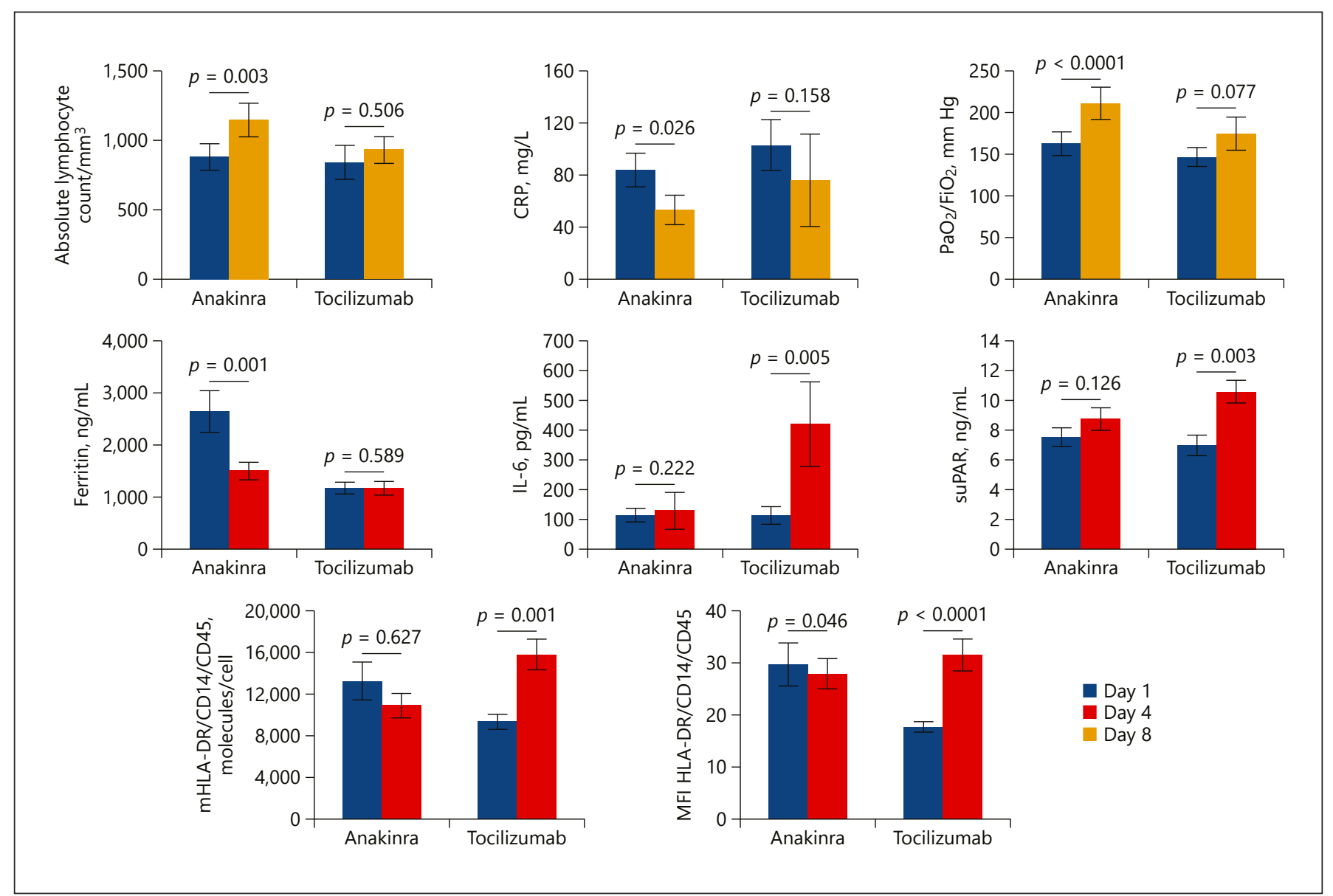

Fig. 2. Change of biomarkers after immunomodulatory treatment for critical COVID-19 patients. Patients were treated with anakinra $(n=60)$ or tocilizumab $(n=42)$. Comparative values of day 1 before start of treatment and of day 8 are provided for the absolute lymphocyte count, serum CRP, and the ratio of partial oxygen pressure to the fraction of inspired oxygen $\left(\mathrm{PaO}_{2} / \mathrm{FiO}_{2}\right)$. Comparative values of day 1 before start of treatment and of day 4 are provided for ferritin, IL-6, suPAR, absolute number of molecules of HLA-DR (mHLA-DR) and MFI of HLA-DR on circulating monocytes. The $p$ values of the respective comparisons by the Wilcoxon paired test are provided. CRP, C-reactive protein; IL, interleukin; HLA, human leukocyte antigen; MFI, mean fluorescence intensity; suPAR, soluble urokinase plasminogen activator receptor. 


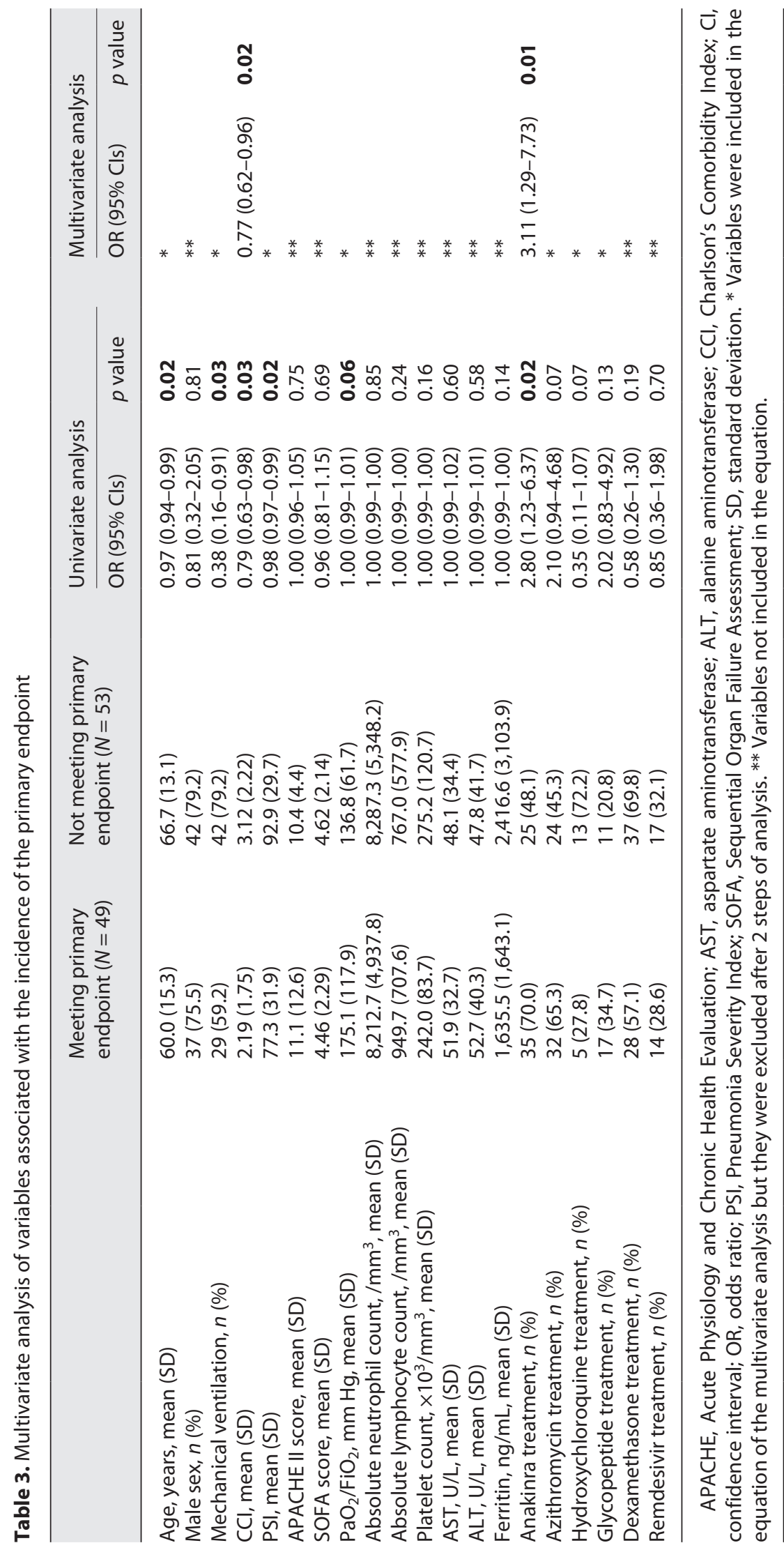




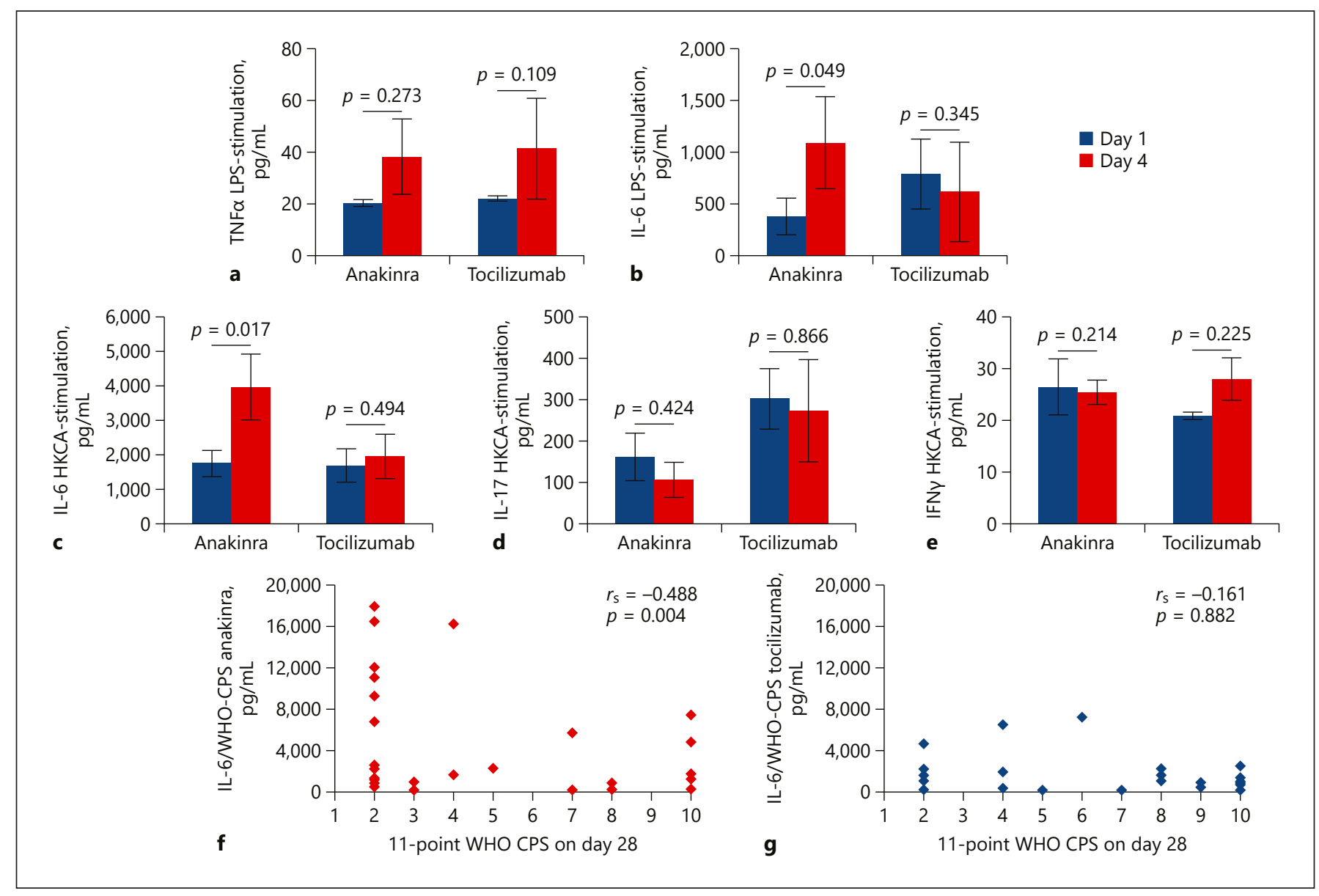

Fig. 3. Modulation of mononuclear cell function (a-e) Concentrations of TNFa, of interleukin (IL)-6, IL-17 and IFN $\gamma$ in the supernatants of PBMCs of patients treated with anakinra and with tocilizumab; PBMCs were isolated before start of treatment (day 1) and on day 4 of treatment and they were stimulated with LPS of Escherichia coli O55:B5 and with HKCA. $p$ values indicate the level of significance after comparisons between days 1 and 4 by the
Wilcoxon test. f, $\mathbf{g}$ Correlation between IL-6 produced by PBMCs on day 4 after stimulation with HKCA and the WHO-CPS on day 28. The Spearman correlation co-efficient $\left(r_{\mathrm{s}}\right)$ and the $p$ values of significance are provided. CPS, clinical progression scale; HKCA, heat-killed Candida albicans; IL, interleukin; IFN, interferon; LPS, lipopolysaccharide; PBMCs, peripheral blood mononuclear cells; TNFa, tumor necrosis factor-alpha. function not making them eligible for tocilizumab are shown in online suppl. Table 1 (online suppl. material).

Immunotherapy was accompanied by significant changes of biomarkers (Fig. 2). By day 8, the absolute lymphocyte counts and the $\mathrm{PaO}_{2} / \mathrm{FiO}_{2}$ ratio were increased only among anakinra-treated patients; serum Creactive protein was decreased in both groups. By day 4 , circulating ferritin was decreased only among anakinratreated patients. Notably, on the same day 4 , circulating IL-6 and soluble urokinase plasminogen activator receptor (suPAR) were increased among tocilizumab-treated patients. Absolute HLA-DR receptors and the mean fluorescence intensity of HLA-DR on circulating CD14- monocytes were also increased among tocilizumabtreated patients.

Analysis of cytokine production capacity by PBMCs showed that the restoration of the cytokine potential capacity by PBMCs by day 4 was associated with better clinical outcome. More precisely, PBMCs of patients treated with anakinra had increased production capacity for IL-6 compared to baseline before start of treatment; this was not found with tocilizumab. It was also found that the better production capacity for IL- 6 by the PBMCs of anakinra-treated patients by day 4 was associated with lower scales of severity of the 28-day 11-point WHO CPS; this was not found with tocilizumab (Fig. 3). 


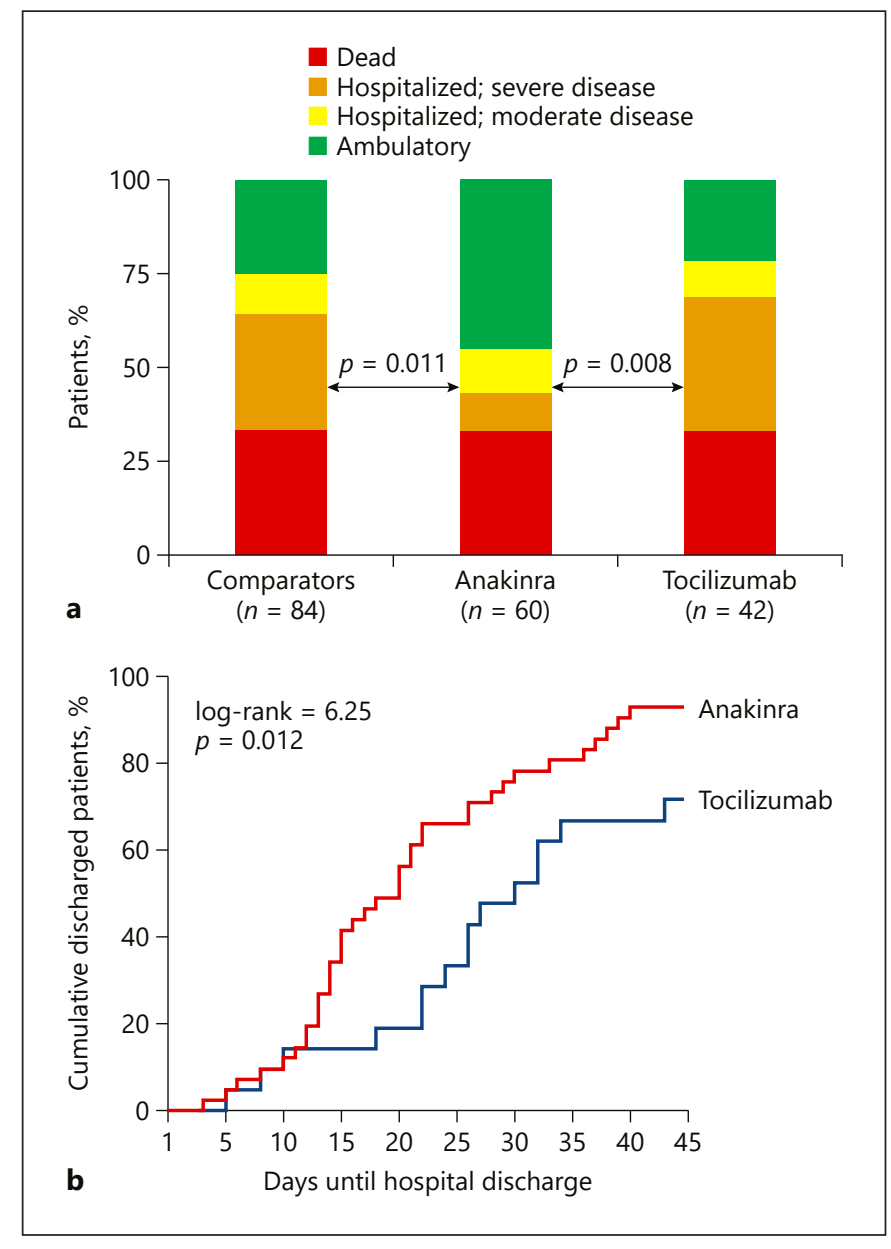

Fig. 4. Exploratory study endpoints. a Comparative distribution of the scales of the WHO clinical progression scale by day 28 between anakinra-treated patients, tocilizumab-treated patients, and available comparators; the indicated comparisons are done by Pearson's $\chi^{2}$ test. $\mathbf{b}$ Time to hospital discharge of patients receiving treatment with anakinra and with tocilizumab. The $p$ value of comparison by the log-rank test is provided.

A total of 84 concurrent comparators were studied; they had the same baseline characteristics as patients enrolled in the ESCAPE trial (online suppl. Table 2, provided in the online suppl. material). Among these comparators, 7 had MALS as defined by serum ferritin $>4,420$ $\mathrm{ng} / \mathrm{mL} ; 6$ of them died by day 28 (85.7\%; 95\% CIs $48.7-$ 97.4\%). Among the 14 patients with MALS treated with anakinra, 9 died by day 28 (64.3\%; 95\% CIs 38.7-83.6\%); 28-day mortality was lower with anakinra treatment ( $p: 0.03$ by the binomial test).

Investigation of the 2 exploratory endpoints - WHO CPS and length of hospital stay - favored anakinra treatment (Fig. 4). More precisely, survivors by day 28 who received anakinra were distributed to less severe scales than patients who received tocilizumab; this superiority of anakinra was also shown versus the concurrent comparators (Fig. 4a). The median time to hospital discharge was 20 days with anakinra treatment and 31 days with tocilizumab treatment (Fig. $4 \mathrm{~b}$ ).

The incidence of secondary infections, particularly pneumonia and bloodstream infections, was greater with tocilizumab treatment. The incidence of aminotransferases increase was also lower with anakinra treatment (Table 4 and online suppl. Table 3, provided in the online suppl. material).

\section{Discussion}

A search at the Clinicaltrials.gov repository of ongoing trials for COVID-19 indicates that ESCAPE is the only trial aiming to deliver immunotherapy to critically ill patients, following a personalized approach. The approach is based on the classification of the immune function of the host, that is, MALS or CID. Although results showed superior efficacy of anakinra compared to tocilizumab for the achievement of the primary endpoint, this should not be interpreted as superiority of anakinra over tocilizumab. The findings suggest that the specific tools used for the classification of MALS and CID most probably favor the use of anakinra over tocilizumab. This treatment effect occurred above and beyond any benefit conferred by concurrent corticosteroid treatment, since the co-administration of dexamethasone was $58 \%$ among anakinra-treated patients and $71 \%$ among patients treated with tocilizumab. Moreover, it is of note that hypertension was more prevalent in the tocilizumab group, but no association was found between the presence of hypertension and failure to achieve the primary endpoint.

ESCAPE was designed in March 2020 and started in April 2020 well before the WHO CPS was introduced as an endpoint for trials of COVID-19. Exploratory application of WHO CPS showed that despite the lack of difference in mortality by day 28 , the allocation of patients treated with anakinra to less severe scales was more frequent than tocilizumab. This is compatible with the earlier discharge of anakinra-treated patients from hospital. The superiority of anakinra was also shown versus matched comparators; anakinra provided survival benefit for patients with MALS. A mechanistic insight on the function of PBMCs was shown for patients treated with anakinra. The improved capacity of PBMCs for the ex vivo production of IL- 6 by day 4 should be considered as restoration of function; it is not associated with a system- 
Table 4. Serious and nonserious adverse events captured during the ESCAPE trial

\begin{tabular}{|c|c|c|c|}
\hline & $\begin{array}{l}\text { Anakinra } \\
(n=60)\end{array}$ & $\begin{array}{l}\text { Tocilizumab } \\
(n=42)\end{array}$ & $p$ value \\
\hline \multicolumn{4}{|l|}{ Serious adverse events, $n(\%)$} \\
\hline Pneumothorax & $2(3.3)$ & $6(14.3)$ & 0.06 \\
\hline Pulmonary embolism & $1(1.7)$ & $0(0)$ & 1.00 \\
\hline Deep venous thrombosis & $1(1.7)$ & $0(0)$ & 1.00 \\
\hline Acute kidney injury & $7(11.7)$ & $5(11.9)$ & 1.00 \\
\hline Shock & $17(28.3)$ & $10(23.8)$ & 0.66 \\
\hline \multicolumn{4}{|l|}{ Infections } \\
\hline Ventilator-associated pneumonia* & $9(15.0)$ & $15(35.7)$ & 0.02 \\
\hline Catheter-related bloodstream infection & $4(6.7)$ & $4(9.5)$ & 0.71 \\
\hline Bloodstream infection** & $13(21.7)$ & $17(40.5)$ & 0.049 \\
\hline Clostridioides difficile infection & $3(5.0)$ & $1(2.4)$ & 0.64 \\
\hline \multicolumn{4}{|l|}{ Arrhythmias } \\
\hline Ventricular tachycardia & $3(5.0)$ & $0(0)$ & 0.27 \\
\hline Atrial fibrillation & $6(10.0)$ & $4(9.5)$ & 1.00 \\
\hline Bradycardia & $0(0)$ & $4(9.5)$ & 0.03 \\
\hline Grade 4 laboratory investigation & $4(6.7)$ & $5(11.9)$ & 0.57 \\
\hline Thrombocytopenia & $3(5.0)$ & $4(9.5)$ & 0.44 \\
\hline Increase of aminotransferases & $0(0)$ & $3(7.1)$ & 0.07 \\
\hline Increase of CPK & $1(1.7)$ & $2(4.8)$ & 0.57 \\
\hline \multicolumn{4}{|l|}{ Nonserious adverse events } \\
\hline Grade $1 / 2$ thrombocytopenia & $3(5.0)$ & $4(9.5)$ & 0.44 \\
\hline Grade $1 / 2$ increase of aminotransferases & $5(8.3)$ & $14(33.3)$ & 0.002 \\
\hline Grade $1 / 2$ increase of creatinine & $6(10.0)$ & $8(19.1)$ & 0.25 \\
\hline Grade $1 / 2$ increase of glucose & $3(5.0)$ & $2(4.8)$ & 1.00 \\
\hline Grade $1 / 2$ hyponatremia & $5(8.3)$ & $4(9.5)$ & 1.00 \\
\hline Grade $1 / 2$ hypokalemia & $1(1.7)$ & $5(11.9)$ & 0.08 \\
\hline Grade $1 / 2$ hyperkalemia & $5(8.3)$ & $4(9.5)$ & 1.00 \\
\hline Grade $1 / 2$ increase of CPK & $0(0)$ & $3(7.1)$ & 0.07 \\
\hline
\end{tabular}

Adverse events were graded according to the Common Terminology Criteria for Adverse Events (version 5.0). Adverse events of grade 4 were considered as serious adverse events. CPK, creatinine phosphokinase. *Pathogens of ventilator-associated pneumonia in the anakinra group were: Acinetobacter baumannii $(n=7)$; Pseudomonas aeruginosa $(n=1)$; and Aspergillus spp. $(n=1)$. Pathogens of ventilator-associated pneumonia in the tocilizumab group were A. baumannii $(n=7)$; P. aeruginosa $(n=3)$; Klebsiella pneumoniae $(n=1)$; Enterobacter cloacae $(n=1)$; Staphylococcus aureus $(n=1)$; and Stenotrophomonas maltophilia $(n=1)$ ( 2 patients of the tocilizumab arm developed ventilator-associated pneumonia by $>1$ pathogens, whereas no pathogen was identified in 3 cases). **Bloodstream pathogens in the anakinra group were: A. baumannii $(n=5)$; Enterococcus faecium $(n=3) ;$ K. pneumoniae $(n=2)$; P. aeruginosa $(n=1)$; Citrobacterfreundii $(n=1)$; Enterobactercloacae $(n=1)$; Enterococcus faecalis $(n=1)$; Candida albicans $(n=1)$ ( 2 patients had $>1$ pathogens). Bloodstream pathogens in the tocilizumab group were A. baumannii $(n=5) ;$. pneumoniae $(n=4)$; E. faecalis $(n=2)$; E. faecium $(n=2)$; P. aeruginosa $(n=2)$; Acinetobacter xylosoxidans $(n=1)$; S. aureus $(n=1)$; and Staphylococcus hominis $(n=1)$ (1 patient had $>1$ pathogen). ic release of IL-6, and it is associated with better clinical outcomes.

Treatment with anakinra decreased serum ferritin, probably indicating attenuation of macrophage activation. The expression of HLA-DR on circulating monocytes was increased with tocilizumab, as previously described [7]. Treatment with tocilizumab was accompanied by an increase in serum IL-6, as described by others [12]. Tocilizumab is an- tagonizing the receptor of IL-6, and this may lead to a reciprocal increase in IL-6. Remarkably, in the same patients, suPAR was also increased, which is a biomarker of poor prognosis $[13,14]$. Whether there is a mechanistic link between the increase in IL- 6 and suPAR and the lack of clinical improvement requires more research.

Important questions remain still unanswered for biological therapies in COVID-19: which is the best candi- 
date patient, for which type of treatment, and what is the correct time frame from disease onset to start the treatment. Retrospective data for the efficacy of intravenous anakinra report clinical efficacy in cohorts ranging from 12 to 65 patients respectively and using concurrent comparators [15-19]. Results suggest that it is better to start anakinra before mechanical ventilation is needed. ESCAPE was conducted in patients with profound organ dysfunction and results proved that anakinra benefit may still be obtained even when COVID-19 has substantially progressed if this is guided by biomarkers. Results corroborate the reported efficacy of intravenous anakinra in 7 mechanically ventilated patients with macrophage activation; the administered dose was $200 \mathrm{mg} 3$ times daily for 7 days and 5 patients were remarkably improved [20].

Results of 3 randomized clinical trials provided contradictory evidence on tocilizumab efficacy to prevent the incidence of mechanical ventilation or death, compared to placebo when given early in hospitalized patients in the ward [21-23]. However, 2 large platform trials, RECOVERY and REMAP-CAP, suggest for significant clinical efficacy in critically ill patients. In the RECOVERY trial, among 4,116 patients with hypoxic COVID-19 and evidence of systemic inflammation, a 28 -day mortality reduction of $4 \%$ was achieved in the tocilizumab group providing a number needed to treat 25 to avoid 1 death [24]. One preliminary report of the REMAP-CAP trial of 895 patients reported $8 \%$ decrease in hospital mortality and days of organ support [25].

The main limitations of the ESCAPE trial are (a) the lack of randomized, double-blind and double-dummy design; (b) the lack of immune assessment for CID in the concurrent comparator group; and (c) the different rate of co-administration of azithromycin, hydroxychloroquine, remdesivir, and dexamethasone between treated patients and concurrent comparators. However, the rate of co-administration of these drugs between the anakinra and tocilizumab arm was similar and cannot affect the primary endpoint.

The findings of the present study suggest clinical benefit among critically ill patients when anakinra treatment is guided by either ferritin $>4,420 \mathrm{ng} / \mathrm{mL}$ which is diagnostic of MALS or with low expression of HLA-DR on CD14-circulating monocytes and increase of aminotransferases. This benefit should be confirmed in a randomized clinical trial.

\section{Acknowledgments}

The manuscript is available as pre-print at medRxiv at https:// medrxiv.org/cgi/content/short/2021.01.20.21250182v1 [26].

\section{Statement of Ethics}

The study was conducted ethically in accordance with the World Medical Association Declaration of Helsinki and was approved by the National Ethics Committee of Greece (reference number 30/20) and the National Organization for Medicines of Greece, IS 021-20. A written informed consent form was provided by all enrolled patients, or their legal representatives.

\section{Conflict of Interest Statement}

George N. Dalekos has acted as advisor/lecturer for Abbvie, Bristol-Myers Squibb, Gilead, Novartis, Roche, Amgen, MSD, Janssen, Ipsen, Genkyotex, Sobi, and Pfizer; has received grant support from Bristol-Myers Squib, Gilead, Roche, Janssen, Abbvie, and Bayer; and was or is currently PI in national and international protocols sponsored by Abbvie, Bristol-Myers Squibb, Novartis, Gilead, Novo Nordisk, Genkyotex, Regulus Therapeutics Inc., Tiziana Life Sciences, Bayer, Astellas, Ipsen, Pfizer, Amyndas Pharamaceuticals, CymaBay Therapeutics Inc., and Roche. E. Karakike has received funding from Horizon $2020 \mathrm{Ma}-$ rie Skłodowska-Curie Grant European Sepsis Academy (Grant No. 676129), outside of the submitted work. Haralampos Milionis reports receiving honoraria, consulting fees, and nonfinancial support from health-care companies, including Amgen, Angelini, Bayer, Mylan, MSD, Pfizer, and Servier. J. Eugen-Olsen is a cofounder, shareholder, and CSO of ViroGates A7S, Denmark, and is named inventor on patents on suPAR owned by Copenhagen University Hospital Hvidovre, Denmark. He is granted by the Horizon 2020 European Grant RISKinCOVID. M.G. Netea is a scientific founder of TTxD and received research grants from GSK and ViiV Healthcare. E.J. Giamarellos-Bourboulis has received honoraria from Abbott $\mathrm{CH}$, Angelini Italy, InflaRx GmbH, MSD Greece, XBiotech Inc., Sobi AB, and B.R.A.H.M.S GmbH (Thermo Fisher Scientific); independent educational grants from AbbVie Inc., Abbott CH, Astellas Pharma Europe, AxisShield, bioMérieux Inc, Johnson \& Johnson, Novartis, InflaRx GmbH, Sobi AB, XBiotech Inc (granted to the Hellenic Institute for the Study of Sepsis); and funding from the FrameWork 7 program HemoSpec (granted to the National and Kapodistrian University of Athens), the Horizon 2020 Marie-Curie Project European Sepsis Academy (granted to the National and Kapodistrian University of Athens), the Horizon 2020 European Grant ImmunoSep (granted to the Hellenic Institute for the Study of Sepsis), and the Horizon 2020 RISKinCOVID (granted to the Hellenic Institute for the Study of Sepsis). The other authors do not report any conflict of interest.

\section{Funding Sources}

This work was supported partly by the Hellenic Institute for the Study of Sepsis and partly by the Horizon 2020 grant RISKinCOVID (Grant No. 961844). The funders had no role in the design, collection, analysis and interpretation of data, writing, and decision to publish. 


\section{Author Contributions}

E.J.G.B. conceptualized the study design, participated in data analysis and drafting the manuscript, had full access to all of the study data, and takes responsibility for their integrity and the accuracy of the analysis. E.J.G.B. and E.K. have verified the underlying data. E.K., J.E.O., and M.G.N. analyzed the data and drafted the manuscript. G.N.D., I.K., M.S., C.P., G.P., A.K., S.C., V.D., N.V., E.P., C.A., A.I., E.K.o., A.K., E.N., N.R., E.S., H.M., G.N., A.K., G.D., V.Z., K.A., N.K.G., V.K., E.G., and N.M. collected clinical data and critically revised the manuscript for important intellec- tual content. G.D.a. performed laboratory measurements and critically revised the manuscript for important intellectual content. All authors had full access to all of the study data and approved the final version of the manuscript to be submitted for publication.

\section{Data Availability Statement}

Online suppl. material associated with this article can be found in the online version.

\section{References}

1 Qin C, Zhou L, Hu Z, Zhang S, Yang S, Tao Y, et al. Dysregulation of immune response in patients with coronavirus 2019 (COVID-19) in Wuhan, China. Clin Infect Dis. 2020 Jul; 71(15):762-8.

2 McElvaney OJ, McEvoy NL, McElvaney OF, Carroll TP, Murphy MP, Dunlea DM, et al. Characterization of the inflammatory response to severe COVID-19 illness. Am J Respir Crit Care Med. 2020 Sep;202(6):81221.

3 Ruscitti P, Berardicurti O, Di Benedetto P, Cipriani P, Iagnocco A, Yehuda Shoenfeld Y, et al. Another piece in the puzzle of the hyperferritinemic syndrome. An immunomodulatory perspective to alleviate the storm. Front Immunol. 2020 May;11:1130.

4 Blot M, Bour JB, Quenot JP, Bourredjem A, Nguyen M, Guy J, et al. The dysregulated innate immune response in severe COVID-19 pneumonia that could drive poorer outcome. J Transl Med. 2020 Dec;18(1):457.

5 Colafrancesco S, Alessandri C, Conti F, Priori R. COVID-19 gone bad: a new character in the spectrum of the hyperferritinemic syndrome? Autoimmun Rev. 2020 Jul;19(7): 102573.

6 Kappert K, Jahić A, Tauber R. Assessment of serum ferritin as a biomarker in COVID-19: bystander or participant? Insights by comparison with other infectious and non-infectious diseases. Biomarkers. 2020 Dec;25(8):616-25.

7 Giamarellos-Bourboulis EJ, Netea MG, Rovina N, Akinosoglou K, Antoniadou A, Antonakos $\mathrm{N}$, et al. Complex immune dysregulation in COVID-19 patients with severe respiratory failure. Cell Host Microbe. 2020 Jun;27(6): 992-1000.

8 Kyriazopoulou E, Leventogiannis K, NorrbyTeglund A, Dimopoulos G, Pantazi A, Orfanos SE, et al. Macrophage activation-like syndrome: an immunological entity associated with rapid progression to death in sepsis. BMC Med. 2017 Sep;15(1):172.

9 Karakike E, Giamarellos-Bourboulis EJ. Macrophage activation-like syndrome: a distinct entity leading to early death in sepsis. Front Immunol. 2019 Jan;10:55.
10 Shakoory B, Carcillo JA, Chatham WW, Amdur RL, Zhao H, Dinarello CA, et al. Interleukin-1 receptor blockade is associated with reduced mortality in sepsis patients with features of macrophage activation syndrome: reanalysis of a prior phase III trial. Crit Care Med. 2016 Feb;44(2):275-81.

11 WHO Working Group on the clinical characterisation and management of COVID-19 infection. A minimal common outcome measure set for COVID-19 clinical research. Lancet Infect Dis. 2020 Aug;20(8):e192-7.

$12 \mathrm{Vu}$ CA, DeRonde KJ, Vega AD, Maxam M, Holt G, Natori Y, et al. Effects of Tocilizumab in COVID-19 patients: a cohort study. BMC Infect Dis. 2020 Dec;20(1):964.

13 Rovina N, Akinosoglou K, Eugen-Olsen J, Hayek S, Reiser J, Giamarellos-Bourboulis EJ. Soluble urokinase plasminogen activator receptor (suPAR) as an early predictor of severe respiratory failure in patients with COVID-19 pneumonia. Crit Care. 2020 Apr;24(1):187.

14 Azam TU, Shadid HR, Blakely P, O'Hayer P, Berlin H, Pan M, et al. Soluble Urokinase Receptor (SuPAR) in COVID-19-Related AKI. J Am Soc Nephrol. 2020 Nov;31(11):2725-35.

15 Cauchois R, Koubi M, Delarbre D, Manet C, Carvelli J, Blasco VB, et al. Early IL-1 receptor blockade in severe inflammatory respiratory failure complicating COVID-19. Proc Natl Acad Sci USA. 2020 Aug;117(32):18951-3.

16 Cavalli G, De Luca G, Campochiaro C, DellaTorre E, Ripa M, Canetti D, et al. Interleukin-1 blockade with high-dose anakinra in patients with COVID-19, acute respiratory distress syndrome, and hyperinflammation: a retrospective cohort study. Lancet Rheumatol. 2020 Jun;2(6):e325-31.

17 Bozzi G, Mangioni D, Minoia F, Aliberti S, Grasselli G, Barbetta L, et al. Anakinra combined with methylprednisolone in patients with severe COVID-19 pneumonia and hyperinflammation: an observational cohort study. J Allergy Clin Immunol. 2021 Feb; 147(2):561-e4.
18 Huet $\mathrm{T}$, Beaussier $\mathrm{H}$, Voisin O, Jouveshomme S, Dauriat G, Lazareth I, et al. Anakinra for severe forms of COVID-19: a cohort study. Lancet Rheumatol. 2020 Jul;2(7):e393-400.

19 Navarro-Millán I, Sattui SE, Lakhanpal A, Zisa D, Siegel CH, Crow MK. Use of anakinra to prevent mechanical ventilation in severe COVID-19: a case series. Arthritis Rheumatol. 2020 Dec;72(12):1990-7.

20 Dimopoulos G, de Mast Q, Markou N, Theodorakopoulou M, Komnos A, Mouktaroudi $\mathrm{M}$, et al. Favorable anakinra responses in severe Covid-19 patients with secondary hemophagocytic lymphohistiocytosis. Cell Host Microbe. 2020 Jul;28(1):117-e1.

21 Hermine O, Mariette X, Tharaux PL, RescheRigon M, Porcher R, Ravaud P, et al. Effect of tocilizumab vs usual care in adults hospitalized with COVID-19 and moderate or severe pneumonia: a randomized clinical trial. JAMA Intern Med. 2021 Jan;181(1):32-40.

22 Stone JH, Frigault MJ, Serling-Boyd NJ, Fernandes AD, Harvey L, Foulkes AS, et al. Efficacy of tocilizumab in patients hospitalized with Covid-19. N Engl J Med. 2020 Dec; 383(24):2333-44.

23 Salama C, Han J, Yau L, Reiss WG, Kramer B, Neidhart JD, et al. Tocilizumab in patients hospitalized with COVID-19 pneumonia. N Engl J Med. 2021 Jan;384(1):20-30.

24 RECOVERY Collaborative Group. Tocilizumab in patients admitted to hospital with COVID-19 (RECOVERY): a randomised, controlled, open-label, platform trial. Lancet. 2021 May 1;397(10285):1637-45.

25 Gordon AC, Mouncey PR, Al-Beidh F, Rowan KM, Nichol AD, et al., REMAP-CAP Investigators. Interleukin-6 receptor antagonists in critically ill patients with Covid-19 interleukin-6 receptor antagonists in critically ill patients with Covid-19. N Engl J Med. 2021 Apr 22;384(16):1491-1502.

26 Karakike E, Dalekos GN, Koutsodimitropoulos I, Saridaki M, Pourzitaki C, Papathanakos G, et al. ESCAPE: an open-label trial of personalized immunotherapy in critically ill COVID-19 patients. MedRxiv. 2021. 